\title{
Nonlinear stochastic dissipation in turbulent geophysical flows
}

\author{
Jean-Michel Brankart \\ Univ. Grenoble Alpes, CNRS, IRD, Grenoble INP, IGE, Grenoble, France \\ Jean-Michel.Brankart@univ-grenoble-alpes.fr
}

This is a non-peer reviewed preprint submitted to EarthArXiv.

It has been submitted on June 18, 2020 to Journal of Advances in Modeling Earth Systems.

\begin{abstract}
The purpose of this study is to further investigate the assumption that geophysical flows can be described and simulated by direct sampling from a dynamically constrained probability distribution, with a reversible formulation of dissipation. This is done by making the probability of the flow decrease with the deviation of the Lagrangian with respect to local equilibrium, and by assuming that this deviation can be computed from the local structure of the macroscopic flow. It is shown that this formalism can reproduce a wide variety of linear dissipation behaviours by adjusting appropriately the spectrum of the stochastic fluctuations in the macroscopic flow, and that it can be easily extended to include nonlinear dissipation in the probability distribution. This reversible, stochastic and nonlinear formulation of dissipation is then applied to the simulation of turbulent geophysical flows, using the lowest-order nonlinear term, with constant parameters. Results obtained for the turbulent boundary layer (in canal flows) show that the main observed characteristics of the flow can be quite faithfully reproduced. In particular, far enough from the boundary conditions, the universal logarithmic velocity profile is an analytic solution of the governing equation, and the behaviour of the friction coefficient as a function of the Reynolds number is correctly simulated by this single model, across the viscous and turbulent regimes. Unlike linear dissipation, the nonlinear dissipation term is specific to the reversible formulation of the problem, and has no equivalent as a forward-in-time diffusion equation. On the other hand, results obtained with a shallow-water model suggest that the same approach can be applied to more complex flows, and that it might become applicable to produce a probabilistic forecast of geophysical flows. More generally, this study suggests that it is possible to construct a reversible probabilistic dynamics that connects observations across time, at a macroscopic level, without explicitly resolving the finest structures of the system.
\end{abstract}

\section{Introduction}

The purpose of this study is to further investigate the assumption that geophysical flows can be described and simulated by direct sampling from a dynamically constrained probability distribution (as proposed in Brankart, 2020). In this framework, the dynamics of the flow, including dissipation, is fundamentally reversible and non-deterministic. Irreversible behaviours only stem from the asymmetry between the initial and final conditions. With prescribed initial condition and free final condition, this formalism can reproduce the same irreversible effect as the classic forward-in-time diffusion equation. On the other hand, stochastic behaviours need not be explicitly simulated by forward-in-time random processes, since they directly result from the random sampling of the probability distribution. 
The main idea that is proposed here to extend this scheme is to introduce nonlinear dissipation in the probability distribution. The range of possible formulation is indeed significantly broader than what can be done with the classic forward-in-time formalism. However, moving away from classic diffusion would ideally also require stabilizing the physical background supporting the probabilistic formalism. This is attempted in this paper by proposing a relation between the stochastic parameterization of dissipation and the underlying statistics of the unresolved scales. The expectation is that the nonlinear formulation of dissipation that is made possible by the reversible probabilistic formalism can be better suited to simulate turbulent geophysical flows, without explicitly resolving the small-scale motions.

The paper is organized as follows. In section 2, the physical background supporting the probabilistic dissipation formalism is presented. In section 3, the classic linear dissipation is reproduced within this formalism and extended towards nonlinear dissipation. This is complemented in annex A by a study of the conditions required to transform the probabilistic formalism into a forward-in-time dissipation equation. In sections 4 and 5, two examples are provided to illustrate the application of nonlinear dissipation to simulate (i) the turbulent boundary layer (in one-dimensional canal flows) and (ii) a turbulent shallow-water flow on a rotating sphere (in two dimensions). This is complemented in annex B by a generalization of the formulation to three-dimensional flows.

\section{From reversible statistics to irreversible flows}

Let the macroscopic flow be described by the fields $Z_{i}(\mathbf{x}, t), i=1, \ldots, n$, defined in the volume $\Omega$, bounded by the external surface $\Sigma$, and between the initial and final time $t_{0}$ and $t_{1}$. The macroscopic fields $Z_{i}$ may include or not the turbulent scales, depending on the definition of the size of the fluid parcels. The $Z_{i}$ should at least include all variables that are necessary to describe the evolution of the state of the system. There can be dynamical variables, such as the components of momentum, veolcity and/or vorticity, and thermodynamical variables, such as internal energy or temperature, concentrations of the constituents of the fluid, etc, depending on the formulation used to describe the reversible behaviour of the macroscopic flow.

\subsection{Deviation of the Lagrangian from equilibrium}

Let us consider a fluid parcel at $\mathbf{x}$ and $t$, and let $\mathcal{L}(\mathbf{x}, t)$ be the Lagrangian of the molecular motion inside the fluid parcel. This Lagrangian is formed using the kinetic energy of the molecular motion relative to the fluid parcel, thus excluding the macroscopic translation and rotation of the fluid parcel, and using the potential energy associated to the interactions between the molecules inside the fluid parcel, thus excluding the potential of the external forces.

The usual approach to describe irreversible flow is to make the assumption of local equilibrium. This means that the statisical distribution describing the molecular motion inside the fluid parcel at $\mathbf{x}$ and $t$ is an equilibrium distribution, as for instance the Maxwell distribution for velocities, and that the characteristics of this distribution only depend on the local value of the macroscopic fields $Z_{i}(\mathbf{x}, t)$. At local equilibrium, the probability distribution of any characteristic of the molecular motion, including $\mathcal{L}(\mathbf{x}, t)$ in particular, is thus completely determined by the local value of the $Z_{i}$.

To move away from local equilibrium, we here make the less restrictive assumption that $\mathcal{L}(\mathbf{x}, t)$ can depend on the local structure of the $Z_{i}$ (in space and time):

$$
\mathcal{L}-\mathcal{L}^{\mathrm{eq}}=\sum_{i=1}^{n} \sum_{j=1}^{n}\left\{\alpha_{i j}^{(1)} \frac{\delta Z_{i}}{\delta t} \frac{\delta Z_{j}}{\delta t}+\alpha_{i j}^{(2)} \frac{\delta^{2} Z_{i}}{\delta t^{2}} \frac{\delta^{2} Z_{j}}{\delta t^{2}}+\beta_{i j}^{(1)} \nabla Z_{i} \cdot \nabla Z_{j}+\beta_{i j}^{(2)} \Delta Z_{i} \Delta Z_{j}\right\}
$$


where $\mathcal{L}^{\mathrm{eq}}$ is the Lagrangian that the molecular motion would have if the fluid parcel was relaxed to local equilibrium. In Eq. (1), the local expansion has been limited to the second derivatives of the $Z_{i}$ and to quadratic functions of these derivatives. The time derivatives $\delta / \delta t$ are meant to include only the residual part of the variations of the $Z_{i}$, when all non-dissipative effects have been taken into account. For example, if $Z_{i}$ is a passive tracer that can be advected, $\delta / \delta t$ must be the material derivative (see section 5 and annex B for other examples). Equation (1) is then the most general formulation that is compatible with the physical symmetry requirements: reversibility in time and invariance with respect to the rotation of the axes. Only the last term could be expanded to include all invariants of $\nabla \nabla Z_{i}$, but this is here reduced to the simple Laplacian to simplify the notations. It must also be noted that, in this framework, the thermodynamic variables can be univoquely defined by the local equilibrium situation from which the deviation of the Lagrangian is computed.

The symmetric matrices $\alpha^{(k)}$ and $\beta^{(k)}$ are phenomenological coefficients describing the behaviour of the fluid. Asymptotically close to local equilibrium, the limit behaviour of these coefficients is to depend on the local value of the $Z_{i}$ only, but again they can be expanded in terms of the derivatives of the $Z_{i}$, for instance:

$$
\beta_{i j}^{(1)}=\beta_{i j}^{(1,1)}+\sum_{k=1}^{n} \sum_{l=1}^{n} \beta_{i j k l}^{(1,2)} \nabla Z_{k} \cdot \nabla Z_{l}+\ldots
$$

This is obviously equivalent to including higher order terms in Eq. (1). These orders of approximation will be interpreted as different behaviour of dissipation. First order dissipation is discussed in section 3. Examples of possible second order behaviours are given in section 4 and 5 .

\subsection{A least action governing principle}

To obtain a governing principle for the macroscopic flow, we assume that the evolutions of the microscopic motion and the macroscopic fields $Z_{i}$ can be described separately. On the one hand, the molecular motion fastly relax to local equilibrium without completely reaching it because of the ever changing structure of the $Z_{i}$ (until global equilibrium is reached). This is just a weakened version of the local equilibrium assumption. On the other hand, the macroscopic fields $Z_{i}(\mathbf{x}, t)$ organize their evolution to minimize action:

$$
S=\int_{t_{0}}^{t_{1}} \int_{\Omega}\left(\mathcal{L}-\mathcal{L}^{\mathrm{eq}}\right) d \Omega d t
$$

This can be seen as a direct application of the least action principle to the global molecular motion, in the whole volume $\Omega$, between $t_{0}$ and $t_{1}$. This is done by considering the deviation with respect to local equilibrium, to which the molecular motion fastly relax (i.e. locally in each fluid parcel, but not globally in $\Omega$ ), and by assuming that the deviation $\mathcal{L}-\mathcal{L}^{\text {eq }}$ can be computed as a function of the macroscopic fields $Z_{i}$ (i.e. that the phenomenological coefficients are known).

However, we must remember that $\mathcal{L}-\mathcal{L}^{\text {eq }}$ is not a deterministic function of the $Z_{i}$, but can display fluctuations depending on the probability distribution for the molecular motion. Therefore, the least action principle in Eq. (3) transforms into a probability distribution for the evolution of the macroscopic fields $Z_{i}(\mathbf{x}, t)$, which we can write:

$$
p\left(Z_{i}\right) \propto \exp \left(-S / S_{0}\right)
$$

where $S_{0}$ is a parameter ruling the spread of the distribution. For small fluctuations, close enough to local equilibrium, only the quadratic terms remains in Eq. (1), so that the probability distribution (4) becomes asymptotically Gaussian, with a time and space covariance structure 
(or power spectrum) determined by the matrices $\alpha^{(k)}$ and $\beta^{(k)}$. These symmetric matrices are thus constrained by the positive definiteness of the covariance structure. In Eq. (1), the fluctuations that are uncorrelated in space and time have been omitted (i.e. there is no term in $Z_{i} Z_{j}$ ) by considering a deviation to local equilibrium rather than global equilibrium, see Annex A for more details about this.

One important feature of the above statistics is that they are reversible in time. A time asymmetry in the behaviour of the macroscopic fields $Z_{i}(\mathbf{x}, t)$ can thus only come from an asymmetry in the boundary conditions at $t_{0}$ and $t_{1}$. If both initial and final conditions are left unspecified (natural boundary conditions), the minimum of $S$ is obtained for constant and uniform fields: $Z_{i}(\mathbf{x}, t)=C_{i}, \forall i$ (in the absence of an external forcing operating through the external boundary $\Sigma$ or through source and sink terms in $\delta / \delta t)$. But if the initial condition $Z_{i}\left(\mathbf{x}, t_{0}\right)$ is specified and the final condition is left unspecified, then the probability distribution $p\left(Z_{i}\right)$ will promote evolutions of the system in which the structures are progressively removed, so that the macroscopic fields $Z_{i}(\mathbf{x}, t)$ are relaxed towards constant and uniform fields (in the absence of an external forcing). In the following, it will be assumed that there is one direction of time in which no condition is specified, and the convention will be that time is counted positively in this direction. With this additional requirement, it will be shown in the next section that the minimum of $S$ is equivalent to the classic diffusion equation, with the same interpretation of the phenomenological coefficients, providing that the problem is purely diffusive $(\delta / \delta t=\partial / \partial t)$.

\section{From linear to nonlinear dissipation}

To make the connection between the assumptions described in the previous section and the classic formulation of the problem, we start by considering diffusion alone (sections 3.1 and 3.2). In this simple situation indeed, the two formulations can be shown equivalent, in the particular case of an initial condition problem. From this situation of equivalence, it is then easier to figure out why the two formulations must diverge if more complex processes are involved (section 3.3).

\subsection{Diffusion equation}

To simplify the problem to linear diffusion alone, we must reduce the deviation of the Lagrangian to a quadratic function of the first-order time derivatives $\left(\alpha_{i j}^{(1)}\right)$ and seond-order space derivatives $\left(\beta_{i j}^{(2)}\right)$, and let dissipation be the only cause of variation of the $Z_{i}(\delta / \delta t=\partial / \partial t)$, so that $S$ can be written:

$$
S=\int_{t_{0}}^{t_{1}} \int_{\Omega}\left[\left(\frac{\partial \mathbf{Z}}{\partial t}\right)^{T} \mathbf{A}^{2}\left(\frac{\partial \mathbf{Z}}{\partial t}\right)+(\Delta \mathbf{Z})^{T} \mathbf{B}^{2}(\Delta \mathbf{Z})\right] d \Omega d t
$$

where $\mathbf{Z}$ is a vector with the macroscopic fields $Z_{i}(\mathbf{x}, t), \mathbf{A}$, the square root of $\alpha^{(1)}$, and $\mathbf{B}$, the square root of $\beta^{(2)}$.

The minimum of $S$ (i.e., the most probable evolution of the system) must satisfy the EulerLagrange equations (Gelfand and Fomin, 1964):

$$
\mathbf{A}^{2} \frac{\partial^{2} \mathbf{Z}}{\partial t^{2}}=\mathbf{B}^{2} \Delta \Delta \mathbf{Z}
$$

or, if the matrices $\mathbf{A}$ and $\mathbf{B}$ commute (a condition of reducibility, see annex $\mathbf{A}$ ):

$$
\left(\mathbf{A} \frac{\partial}{\partial t}-\mathbf{B} \Delta\right)\left(\mathbf{A} \frac{\partial \mathbf{Z}}{\partial t}+\mathbf{B} \Delta \mathbf{Z}\right)=0
$$

In the particular case of an initial condition problem, i.e., with a natural final condition resulting from the minimization of (5), Eq. (7) can be simplified to: 


$$
\mathbf{A} \frac{\partial \mathbf{Z}}{\partial t}=\mathbf{B} \Delta \mathbf{Z} \quad \text { or } \quad \frac{\partial \mathbf{Z}}{\partial t}=\left(\mathbf{A}^{-1} \mathbf{B}\right) \Delta \mathbf{Z}
$$

This deduction of Eqs. (7) and (8) from the variational principle in (5) is a direct generalization of what was done in Brankart (2020) for a single tracer field.

Equation (8) is equivalent to the classic diffusion equation if $\boldsymbol{\Lambda}=\mathbf{A}^{-1} \mathbf{B}$ is the matrix with the diffusion coefficients. In this particular case, diffusion is operating forward in time because conditions are imposed on the initial condition, whereas the future of the system is left unconstrained. In the expression of $S$ in Eq. (5), however, past and future are symmetric, so that diffusion could, in principle, operate in the two directions of time, as clearly displayed in Eq. (7).

\subsection{Generalized spectrum of fluctuations}

In this formalism, a first route to generalize the description of dissipation is to complexify the time and space covariance structure of the fluctuations. To stay in a linear dissipation framework, we just include more quadratic terms in Eq. (5), with higher-order derivatives of $\mathbf{Z}$. For instance, for only one macroscopic field $Z(n=1)$ and one dimension of space $x$, Eq. (5) would genreralize to:

$$
S=\int_{t_{0}}^{t_{1}} \int_{x_{0}}^{x_{1}}\left[\sum_{k=0}^{K} \alpha^{(k)}\left(\frac{\partial^{k} Z}{\partial t^{k}}\right)^{2}+\sum_{l=0}^{L} \beta^{(l)}\left(\frac{\partial^{l} Z}{\partial x^{l}}\right)^{2}\right] d x d t
$$

In this equation, we keep the terms $k=0$ and $l=0$ (which coincide) for generality (as in annex A), but they must disappear with the assumption that the system is close to local equilibrium, as explained in section 2. The generalization to more fields $Z_{i}$ and to more spatial dimensions is straightforward, but would just make the following equations and explanations more intricate.

The Euler-Lagrange equation corresponding to the variational principle in (9) is:

$$
\sum_{k=0}^{K}(-1)^{k} \alpha^{(k)} \frac{\partial^{2 k} Z}{\partial t^{2 k}}+\sum_{l=0}^{L}(-1)^{l} \beta^{(l)} \frac{\partial^{2 l} Z}{\partial x^{2 l}}=0
$$

The higher-order derivatives can be interpreted as different behaviours of dissipation, even if this equation cannot always be simplified into a forward-in-time diffusion operator, as Eq. (8) from Eq. (6). A higher-order time derivative is needed for instance to avoid instantaneous propagation of information through the fluid, which comes as a consequence of keeping only a first-order time derivative in the diffusion equation (8). On the other hand, higher-order space derivatives are sometimes used in geophysical applications to simulate a diffusion with a more elaborate filtering effect. For instance, keeping only $\alpha^{(1)}$ and $\beta^{(4)}$ in Eq. (10) would lead to a biharmonic dissipation operator (e.g. Delhez and Deleersnijder, 2007).

These various behaviours of dissipation are related to different spectra of the fluctuations. Equation (9) can indeed be reformulated in terms of the Fourier transform $\tilde{Z}(\kappa, \omega)$ of $Z(x, t)$ :

$$
S=\iint \tilde{Z} \tilde{Z}^{*}\left(\sum_{k=0}^{K} \alpha^{(k)} \omega^{2 k}+\sum_{l=0}^{L} \beta^{(l)} \kappa^{2 l}\right) d \kappa d \omega
$$

where $\omega$ and $\kappa$ are the frequency and the wave number. This transformation required extending the bounds of the integral to infinity and applying the Parseval theorem. If the field $Z(x, t)$ is constrained at $t=0$, with the spectrum:

$$
\tilde{Z}_{0}(\kappa)=\int \tilde{Z}(\kappa, \omega) d \omega
$$

the minimum of $S$ is given by: 


$$
\tilde{Z}(\kappa, \omega)=\frac{g(\kappa)}{\sum_{k=0}^{K} \alpha^{(k)} \omega^{2 k}+\sum_{l=0}^{L} \beta^{(l)} \kappa^{2 l}}
$$

where the function $g(\kappa)$ is such that condition (12) is satisfied. This corresponds to a time and space filtering of the condition imposed at $t=0$. The information given by the constraint is forgotten symmetrically in time (for increasing $|t|$ ), according to the time and space spectrum of the fluctuations.

\subsection{Nonlinear effects}

Two types of nonlinear behaviours of the flow must be distinguished: (i) those directly resulting from nonlinear macroscopic effects, and (ii) those resulting from the nonlinear relaxation of the system towards local equilibrium of the microscopic distribution. The separation between these two types of behaviours depends on the scale used to define the size of the fluid parcels.

Macroscopic trends. As explained in section 2, the time derivatives in Eq. (1) are meant to include only the variations of the $Z_{i}$ that results from the relaxation of the flow towards local equilibrium. All deterministic macroscopic trends must be included in the definition of $\delta / \delta t$. For instance, in presence of advection, $\delta / \delta t$ must be the material derivative $D / D t$, so that the macroscopic movement of the fluid parcels is taken into account before considering the residual trend associated to the stochastic dissipation. In this case, material derivatives must replace the partial time derivatives in the expression of $S$ in Eq. (5), and the associated Euler-Lagrange equations in Eq. (6) transforms to:

$$
\mathbf{A}^{2} \frac{D^{2} \mathbf{Z}}{D t^{2}}=\mathbf{B}^{2} \Delta \Delta \mathbf{Z}
$$

as a direct generalization of what was done in Brankart (2020) for a single tracer field. In contrast with the linear Eq. (6), this nonlinear equation cannot be simplified to the classic advection/diffusion equation in the particular case of an initial condition problem. Assuming that the statistics of the fluctuations are the same in the presence of advection (as done here) is thus not equivalent to keeping the same maximum probability diffusive flux (as in the classic advection/diffusion equation). This is an important difference because this deprives us from the possibility of computing the flow as the solution of a forward-in-time differential problem (see Brankart, 2020, for more details about this).

More sophisticated examples of $\delta / \delta t$, including the joint effect of advection, pressure gradient and rotation (through the Coriolis force) are given in section 5 and in annex B. Another important effect to include in $\delta / \delta t$ is the trend in the thermodynamic variables (describing the local equilibrium distribution) resulting from the conversion of energy from the macroscopic flow, but this is out of the scope of the present paper.

Nonlinear dissipation. In the formalism presented in section 2, nonlinear behaviours of the flow can also be obtained by including higher-order terms in the description of dissipation, as illustrated in Eq. (2). As explained in annex A, the variety of possible nonlinear dissipation models is here wider than could be possible with a forward-in-time differential equation, even if they are constrained by reciprocity conditions stemming from the symmetry of the phenomenological coefficients. Two example applications of such a nonlinear dissipation model are discussed in sections 4 and 5. Both suggest that this approach might provide an appropriate stochastic framework to improve the description of turbulent geophysical flows. 


\section{Turbulent boundary layer}

As a first possible application of the nonlinear dissipation framework introduced in the previous section, we consider here the problem of a one-dimensional flow of a viscous fluid between two parallel planes (Poiseuille and Couette flows). The turbulent boundary layer is thus going to be studied in the simplified context of a steady mean flow, with established turbulent regime. In this case, the system can be described by only one macroscopic field $Z_{1}$, which corresponds to the velocity of the fluid $u$, parallel to the planes and this velocity only depends on the distance $x$ with respect to one of the planes: $u=u(x), 0<x<L$, where $L$ is the distance between the two planes. Possible turbulent motions are assumed unresolved, and thus included in the statistical description of the microscopic motion. The expectation is that their effect on the macroscopic flow can be described by adding nonlinear dissipation terms in Eq. (5).

For this problem, Eqs. (1-3) can be simplified to:

$$
S[u]=\int_{t_{0}}^{t_{1}} \int_{0}^{L}\left[\alpha^{(1)}\left(\frac{\delta u}{\delta t}\right)^{2}+\beta^{(1)}\left(\frac{\partial u}{\partial x}\right)^{2}+\beta^{(2)}\left(\frac{\partial^{2} u}{\partial x^{2}}\right)^{2}\right] d x d t
$$

with

$$
\beta^{(1)}=\beta^{(1,0)}+\beta^{(1,1)}\left(\frac{\partial u}{\partial x}\right)^{2} \text { and } \beta^{(2)}=\beta^{(2,0)}+\beta^{(2,1)}\left(\frac{\partial u}{\partial x}\right)^{2}
$$

where we kept only the first two terms in the developments. To further simplify the problem, we first drop the $\alpha^{(1)}$ term, by considering a stationary flow, and we keep only the $\beta^{(2,0)}$ and $\beta^{(1,1)}$ terms. These two terms scale respectively as $U^{2} / L^{4}$ and $U^{4} / L^{4}$, where $U$ is the maximum velocity of the flow. The first one represents the linear viscous dissipation and can be expected to be dominant for small $U$, and the second one is the first nonlinear term that could be introduced in the development.

By adimensionalizing $u$ and $x(\hat{u}=u / U$ and $\hat{x}=x / L)$ and noting the derivatives using indices, Eq. (15) can then be rewritten:

$$
S[\hat{u}]=\int_{0}^{1}\left[\hat{u}_{\hat{x} \hat{x}}^{2}+\gamma^{2} \hat{u}_{\hat{x}}^{4}\right] d \hat{x}
$$

or, in terms of the velocity gradient $\hat{g}=\hat{u}_{\hat{x}}=\partial \hat{u} / \partial \hat{x}$ :

$$
S[\hat{g}]=\int_{0}^{1}\left[\hat{g}_{\hat{x}}^{2}+\gamma^{2} \hat{g}^{4}\right] d \hat{x}
$$

Eqs. (17) and (18) determine the probability distribution of the one-dimensional flow between the two planes. See annex B for a generalization of this formulation to instationary threedimensional flows.

\subsection{Maximum probability velocity profile}

The flow maximizing (17) and (18) must be solution of the corresponding Euler-Lagrange equations (Gelfand and Fomin, 1964):

$$
\hat{u}_{\hat{x} \hat{x} \hat{x} \hat{x}}=6 \gamma^{2} \hat{u}_{\hat{x}}^{2} \hat{u}_{\hat{x} \hat{x}}
$$

or

$$
\hat{g}_{\hat{x} \hat{x}}=2 \gamma^{2} \hat{g}^{3}
$$

with the natural boundary conditions at $\hat{x}=0$ and $\hat{x}=1$ : 


$$
\begin{aligned}
& \hat{u}_{\hat{x} \hat{x} \hat{x}}=2 \gamma^{2} \hat{u}_{\hat{x}}^{3} \\
& \hat{u}_{\hat{x} \hat{x}}=0
\end{aligned}
$$

or

$$
\hat{g}_{\hat{x}}=0
$$

With these conditions, the solution of the problem is then $\hat{g}=0$ or $\hat{u}=$ cst, which corresponds to $S[\hat{u}]=0$ and $S[\hat{g}]=0$.

Essential boundary conditions. In the following, these natural conditions are replaced by essential physical conditions describing the interaction between the fluid and the wall planes:

$$
\hat{u}(0)=\hat{u}(1)=0 \quad \text { and } \quad \hat{g}(0)=-\hat{g}(1)=\hat{g}_{0}
$$

for a Poiseuille flow, or

$$
\hat{u}(1 / 2)=0 \quad \text { and } \quad \hat{g}(0)=\hat{g}(1)=\hat{g}_{0}
$$

for a Couette flow (for which we assumed a zero velocity at $\hat{x}=1 / 2$, to obtain as similar formulation of the boundary layer for the two flows).

First integral. Since $S[g]$ does not explicitly depend on $x$, a first integral of the EulerLagrange equation (20) can be directly obtained (Gelfand and Fomin, 1964):

$$
\hat{g}_{\hat{x}}^{2}=Q+\gamma^{2} \hat{g}^{4} \quad \text { with } \quad \hat{g}=\hat{u}_{\hat{x}}
$$

where $Q$ is an integration constant that must be determined from the boundary conditions. In view of the symmetry of the problem, it can be interpreted as $Q=\hat{g}_{\hat{x}}^{2}(1 / 2)=\hat{u}_{\hat{x} \hat{x}}^{2}(1 / 2)$ for the Poiseuille flow (since $\hat{g}(1 / 2)=\hat{u}_{\hat{x}}(1 / 2)=0$ ), and as $Q=-\gamma^{2} \hat{g}^{4}(1 / 2)=-\gamma^{2} \hat{u}_{\hat{x}}^{4}(1 / 2)$ for the Couette flow ( provides a complete description of the maximum probability velocity profile that we are looking for.

Viscous flow. At the linear limit, for small velocities, when the term with coefficient $\gamma$ is negligible, Eq. (26) reduces to:

$$
\hat{u}_{\hat{x} \hat{x}}^{2}=Q
$$

which directly provides the classic viscous Poiseuille velocity profile:

$$
\hat{u}=\hat{g}_{0} \hat{x}(1-\hat{x})=4 \hat{x}(1-\hat{x})
$$

with boundary conditions (24), and the Couette velocity profile:

$$
\hat{u}=\hat{g}_{0}\left(\hat{x}-\frac{1}{2}\right)=2\left(\hat{x}-\frac{1}{2}\right)
$$

with boundary conditions (25). In both cases, the value of $\hat{g}_{0}$ is provided by the normalizing condition resulting from adimensionalization (with the maximum velocity of the flow). 
Numerical integration. With the nonlinear terms, we can rewrite Eq. (26) in a differential form by separating the variations of $\hat{g}$ and $\hat{x}$ :

$$
\frac{d \hat{g}}{\sqrt{Q+\gamma^{2} \hat{g}^{4}}}= \pm d \hat{x}
$$

which must be integrated a first time to obtain $\hat{g}(\hat{x})$ and a second time to obtain $\hat{u}(\hat{x})$. The sign of the second member of Eq. (30) depends on the sign of $\hat{g}_{\hat{x}}$, which is negative in both flow for $0<\hat{x}<1 / 2$ (if $\hat{g}_{0}>0$ ) and positive only in the Couette flow for $1 / 2<\hat{x}<1$ (if $\hat{g}_{0}>0$ ). By symmetry of the problem, we only compute the solution on the interval $0<\hat{x}<1 / 2$ so that we always keep the negative sign in Eq. (30) and we must replace the boundary condition at $\hat{x}=1$ by the symmetry condition:

$$
\hat{g}(1 / 2)=0
$$

for the Poiseuille flow (with $Q>0$ ), or

$$
\hat{g}_{\hat{x}}(1 / 2)=0 \Rightarrow \hat{g}(1 / 2)=\left(\frac{-Q}{\gamma^{2}}\right)^{1 / 4}
$$

for the Couette flow (with $Q<0$ ). Numerically integrating Eq. (30) for various values of $\gamma$ (see section 4.2 for a physical interpretation of this parameter), we obtain the result displayed in Fig. 1. For small $\gamma$, the velocity profile is the one given by Eq. (28) or (29), whereas, for larger $\gamma$, the nonlinear term penalizing the velocity gradient in Eqs. (17) and (18) make the profile flatter with steep boundary layers to meet the wall boundary conditions. Far enough from the linear behaviour, the profile can thus be separated into two regions: (i) an external region with small velocity gradient, with $\gamma^{2} \hat{g}^{4} \ll Q$ (Poiseuille flow) or $\gamma^{2} \hat{g}^{4} \gtrsim Q$ (Couette flow), and (ii) an internal region where the velocity gradient become high enough to neglect the first term of the denominator $\left(\gamma^{2} \hat{g}^{4} \gg Q\right)$.
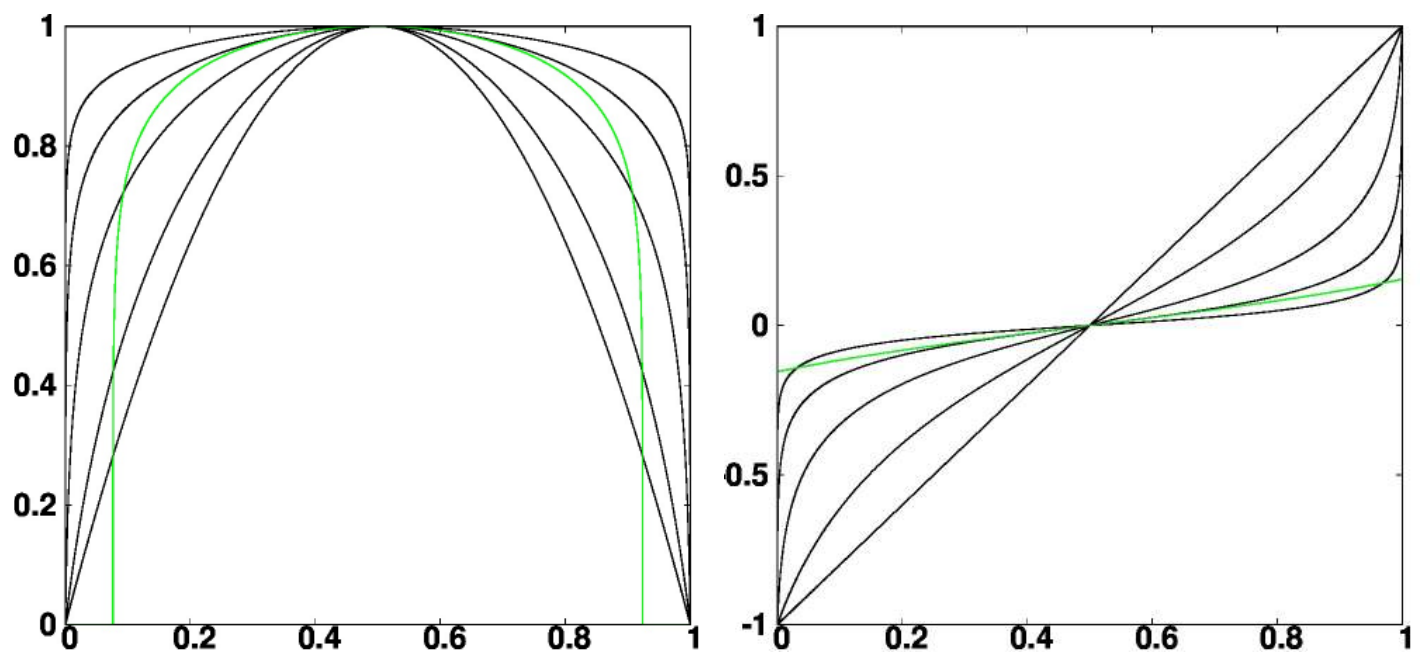

Figure 1: Poiseuille (left panel) and Couette (right panel) velocity profile (black curves) for $\gamma=0,2,5,10,20$ and $\psi=0$. Velocity $\hat{u}$ (y-axis) is drawn as a function of $\hat{x}$ (x-axis). The larger $\gamma$, the steeper the boundary layer. The green curve represents the approximate external velocity profile of Eq. (37) or (39) for $\gamma=10$.

External profile. The external profile can be found by integrating (30) from the external boundary condition (31) or (32): 


$$
\int_{\hat{g}(\hat{x})}^{\hat{g}(1 / 2)} \frac{d \hat{g}}{\sqrt{Q+\gamma^{2} \hat{g}^{4}}}=\hat{x}-\frac{1}{2}
$$

The shape of the solution is thus here different for the Poiseuille flow $(Q>0)$ and the Couette flow $(Q<0)$. In both cases, the solution can be computed (Abramowitz and Stegun, 1970, formulas 17.4.54 and 17.4.55) in terms of an elliptic integral of the first kind $F(\phi \mid 1 / 2)$ :

- Poiseuille flow:

$$
\frac{1}{2} F\left[2 \arctan \left(\theta_{1} \hat{g}\right), \frac{1}{2}\right]=\theta_{2}\left(\frac{1}{2}-\hat{x}\right)
$$

- Couette flow:

$$
\frac{1}{\sqrt{2}} F\left[2 \arccos \left(\frac{1}{\theta_{1} \hat{g}}\right), \frac{1}{2}\right]=\theta_{2}\left(\frac{1}{2}-\hat{x}\right)
$$

with $\theta_{1}^{2}=\gamma / \sqrt{|Q|}$ and $\theta_{2}^{2}=\gamma \sqrt{|Q|}$. Evaluated at $\hat{x}=0$, these equations make the relation between $\hat{g}_{0}$ and $Q$.

To obtain an explicit solution, we can make the approximation $F(\phi \mid 1 / 2) \sim \phi$, with a relative error growing from 0 for $\phi \sim 0$ to about $20 \%$ for $\phi=\pi / 2$. The error can thus be expected to be small where the velocity gradient is small (close to $\hat{x}=\frac{1}{2}$ ) and to become large for increasing $\hat{g}$. With this approximation, the external velocity profile can be computed explicitly:

- Poiseuille flow:

$$
\begin{gathered}
\hat{g}_{\mathrm{ext}}^{P} \simeq \frac{1}{\theta_{1}} \tan \left[\theta_{2}\left(\frac{1}{2}-\hat{x}\right)\right] \\
\hat{u}_{\mathrm{ext}}^{P} \simeq 1+\frac{1}{\gamma} \ln \left\{\cos \left[\theta_{2}\left(\frac{1}{2}-\hat{x}\right)\right]\right\}
\end{gathered}
$$

- Couette flow:

$$
\begin{gathered}
\hat{g}_{\mathrm{ext}}^{C} \simeq \frac{1}{\theta_{1}} \sec \left[\frac{\theta_{2}}{\sqrt{2}}\left(\frac{1}{2}-\hat{x}\right)\right] \\
\hat{u}_{\mathrm{ext}}^{C} \simeq \frac{\sqrt{2}}{\gamma} \ln \left\{\tan \left[\frac{\pi}{4}-\frac{\theta_{2}}{\sqrt{2}}\left(\frac{1}{2}-\hat{x}\right)\right]\right\}
\end{gathered}
$$

In this approximate solution, the integration constant $Q$ depends on the internal condition and the integration constant resulting from the computation of $\hat{u}$ from $\hat{g}$ was chosen so that $\hat{u}_{\text {ext }}^{P}(1 / 2)=1$ and $\hat{u}_{\text {ext }}^{C}(1 / 2)=0$. This approximate external profile is illustrated in Fig. 1 for $\gamma=10$ (green curve), showing that it becomes asymptotically close to the complete numerical solution (black curve) for $\hat{x} \rightarrow 1 / 2$.

Internal profile $\left(\gamma^{2} \hat{g}^{4} \gg Q\right)$. The internal profile can be found by integrating (30) from the internal boundary condition (24) or (25):

$$
\int_{\hat{g}_{0}}^{\hat{g}(\hat{x})} \frac{d \hat{g}}{\hat{g}^{2}}=-\gamma \hat{x}
$$

The shape of the solution is here the same for the Poiseuille and the Couette flows. In both cases, the velocity gradient is given by:

$$
\hat{g}_{\text {int }}(\hat{x})=\frac{1}{\frac{1}{\hat{g}_{0}}+\gamma \hat{x}} \sim \frac{1}{\gamma \hat{x}} \quad \text { for } \quad \hat{x} \gg \frac{1}{\gamma \hat{g}_{0}}
$$

and the velocity profile, by: 


$$
\hat{u}_{\text {int }}(\hat{x})=\frac{1}{\gamma} \ln \left(\gamma \hat{g}_{0} \hat{x}+1\right)
$$

which is approximately logarithmic, far enough from the wall:

$$
\hat{u}_{\text {int }}(\hat{x}) \sim \frac{1}{\gamma} \ln \left(\gamma \hat{g}_{0} \hat{x}\right) \quad \text { for } \quad \hat{x} \gg \frac{1}{\gamma \hat{g}_{0}}
$$

and linear, close to the wall:

$$
\hat{u}_{\text {int }}(\hat{x}) \sim \hat{g}_{0} \hat{x} \quad \text { for } \quad \hat{x} \ll \frac{1}{\gamma \hat{g}_{0}}
$$

In this internal solution, $\hat{g}_{0}$ is a remaining unknown that is constrained by the normalizing condition on the maximum velocity.
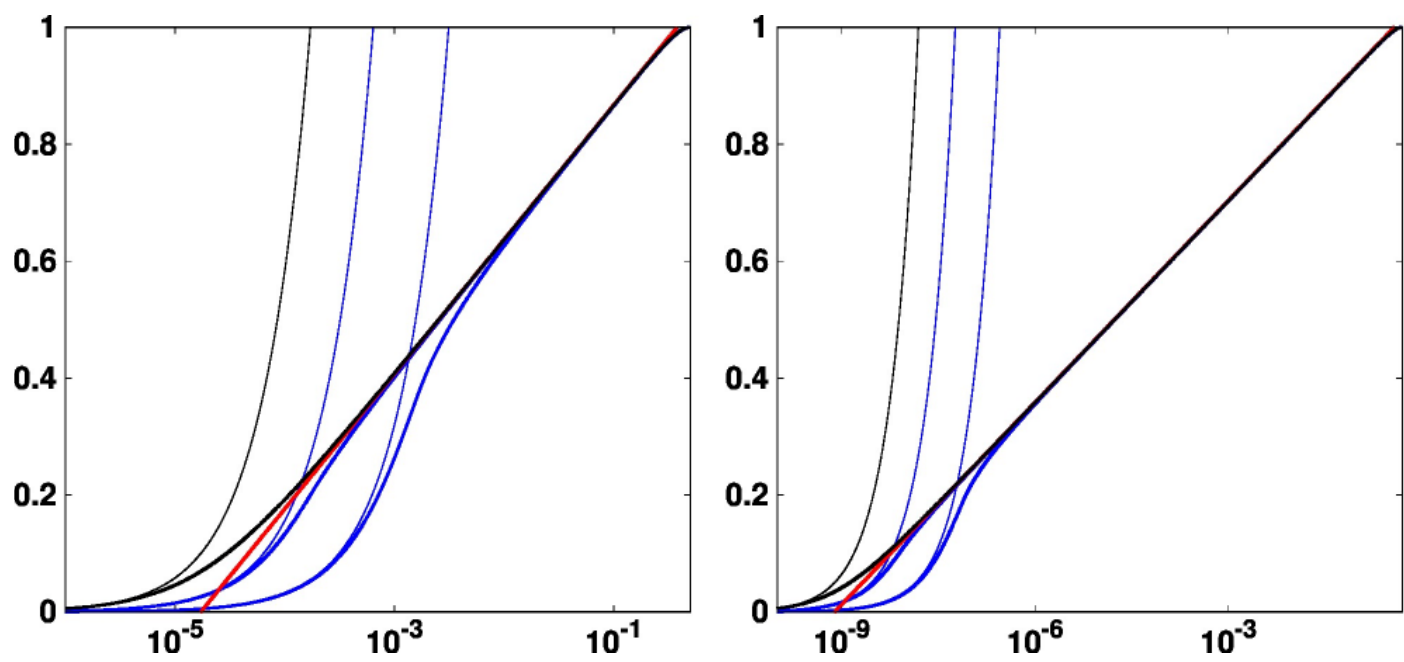

Figure 2: Poiseuille velocity profile with logarithmic x-axis for $\gamma=10$ (left panel) and $\gamma=20$ (right panel), with $\psi=0$ (black curve), $\psi=3$, and $\psi=6$ (blue curves). Velocity $\hat{u}$ (y-axis) is drawn as a function of $\hat{x}$ (x-axis). The larger $\psi$, the thicker the viscous sublayer. All profiles fit a linear profile (thin curves) close to the wall, and the logarithmic profile (thick red curve) over most of the remaining velocity range.

To better see the shape of this internal solution, the velocity profile is displayed in Fig. 2 (thick black curve) with a logarithmic x-axis for $\gamma=10$ (left panel) and $\gamma=20$ (right panel). The profile is linear close to the wall (thin black curve), and becomes quickly logarithmic (thick red curve) over most of the remaining velocity range, except in the external region (close to $\hat{x}=1 / 2$ ).

It must be noted that, disregarding the boundary conditions, the logarithmic velocity profile in Eq. (43) is an exact solution of the Euler-Lagrange equations (19), and corresponds to an exact balance between the two terms of the variational principle (17).

Viscous sublayer. To give more freedom to the behaviour of the flow close to the wall, we can make the additional assumption that $\gamma=0$ in a viscous sublayer of thickness $\hat{\ell}$ given by:

$$
\hat{\ell}=\frac{\psi}{\gamma \hat{g}_{0}}
$$

where $\psi$ is a constant parameter. With a piecewise constant parameter $\gamma$ in Eq. (18), the Euler-Lagrange equations keep the same form, and we only need to apply continuity conditions at the interface between the two regions $(\hat{x}<\hat{\ell}$ and $\hat{x}>\hat{\ell})$. 
The effect of this parameterization is illustrated in Fig. 2 (blue curves) for $\psi=3$ and $\psi=6$. With increasing $\psi$, the linear region becomes thicker and the wall velocity gradient decreases.

\subsection{Fit to observations}

The results obtained in the previous section are all deduced from the variational principle in Eq. (17), with boundary conditions in Eqs. (24) or (25), with a constant or piecewise constant $\gamma$ parameter. The theoretical framework from which they derive can thus be tested by comparing the resulting properties of the velocity profile to the available experimental data.

Logarithmic velocity profile. The most important experimental result about turbulent boundary layers is certainly the observation that, far enough from the outer conditions and far enough from the wall, the velocity profile takes the universal form (Hinze, 1959; Schlichting, 1979):

$$
u^{+}=\frac{1}{\chi} \ln x^{+}+\psi^{+}
$$

where $u^{+}$and $x^{+}$are adimensional variables defined from the dimensional $u$ and $x$ by:

$$
u^{+}=\frac{u}{u_{*}} \quad \text { and } \quad x^{+}=\frac{x u_{*}}{\nu}
$$

and $\chi$ (von Karman constant) and $\psi^{+}$are empirical constants:

$$
\chi \simeq 0.41 \text { and } \psi^{+} \simeq 5.0
$$

In the definition (47), $\nu$ is the kinematic viscosity of the fluid and $u_{*}$ is the shear velocity or friction velocity, which is experimentally obtained from the wall shear stress $\tau_{w}: u_{*}=\sqrt{\tau_{w} / \rho}$. With these definitions, the experimental velocity profile is linear close to the wall with a unit slope: $u^{+}=x^{+}$.

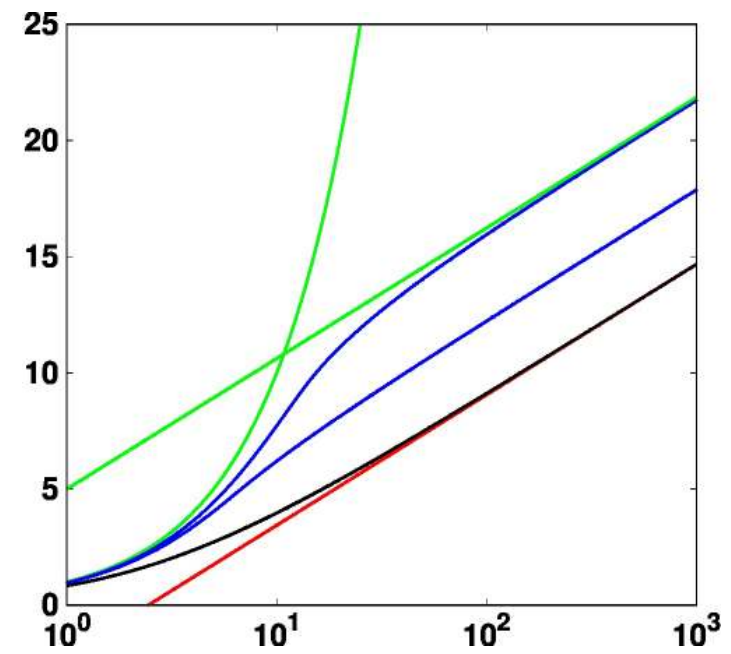

Figure 3: Transformation of Fig. 2 from variables $\hat{x}, \hat{u}$ to variables $u^{+}, x^{+}$. Transformed velocity $u^{+}$(y-axis) is drawn as a function of $x^{+}$(x-axis). The two panels of Fig. 2 transforms into a single graph, with the same linear wall limit $\left(u^{+}=x^{+}\right.$, green curve) and the same logarithmic slope $(1 / \chi)$. In these transformed variables, the effect of increasing $\psi$ is to shift the logarithmic profile. The profile obtained with $\psi=6$ (upper blue curve) is closest to the empirical universal profile, with $\psi^{+}=5$ (green straight line).

To compare this empirical velocity profile with the logarithmic and linear profiles obtained in Eqs. (43) and (44), we must be able to compute $u^{+}$and $x^{+}$from $\hat{u}$ and $\hat{x}$. To do this, we 
must observe first that the empirical and theoretical profiles will display the same logarithmic slope if the parameter $\gamma$ is related to the friction velocity by:

$$
\gamma=\chi \frac{U}{u_{*}}=\chi \hat{u}_{*}^{-1}
$$

Second, from the two expressions of the wall shear stress:

$$
\frac{\tau_{w}}{\rho}=\nu g_{0}=u_{*}^{2},
$$

we obtain the walue of $\nu$ as a function of $u_{*}$ and $g_{0}$, and thus the expression of the Reynolds number (Re), as a function of $\gamma$ and $\hat{g}_{0}$ :

$$
\operatorname{Re}=\hat{\nu}^{-1}=\frac{\hat{g}_{0} \gamma^{2}}{\chi^{2}}
$$

In these formulas, the wall velocity gradient $\hat{g}_{0}$ is obtained from the numerical computation of the velocity profile for any given $\gamma$ and $\psi$. It can thus be expected to depend on specific behaviours of the viscous sublayer, which is here simply described by the constant $\psi$ in Eq. (45).

From Eqs. (49) and (51), we deduce the transformation of $\hat{u}$ and $\hat{x}$ to $u^{+}$and $x^{+}$:

$$
u^{+}=\frac{\gamma}{\chi} \hat{u} \quad \text { and } \quad x^{+}=\frac{\hat{g}_{0} \gamma}{\chi} \hat{x}
$$

so that we can redraw Fig. 2 in terms of $u^{+}$and $x^{+}$instead of $\hat{u}$ and $\hat{x}$. The result is almost identical for $\gamma=10$ and $\gamma=20$, so that only one is shown in Fig. 3. After transformation, all computed profiles (in black an blue) have the same unit slope close to the wall (fitting the green curve: $u^{+}=x^{+}$) and the same logarithmic slope for large $x^{+}$and $u^{+}$(the same as the universal logarithmic profile in green). But the logarithmic part of the profiles are shifted from each other, as a result of the rescaling of $\hat{x}$ by $\hat{g}_{0}$, which depends on the value of $\psi$. For $\psi=0$ (black curve), the wall gradient $\hat{g}_{0}$, is much too large to match the constant $\psi^{+} \simeq 5.0$ of the empirical logarithmic profile (in green). The best match is here obtained for $\psi=6$ (upper blue curve), which makes an appropriate connection between the empirical linear and logarithmic profiles (in green). A specific parameterization of the behaviour of the fluid in the viscous sublayer is thus necessary to fit the constant $\psi^{+}$of the experimental profile.

Friction coefficient. From Eqs. (49) and (51), we can derive the expression of the friction coefficient $C_{f}$ (i) as a function of $\gamma$, and (ii) as a function of Re:

$$
C_{f}=\frac{\tau_{w}}{\frac{1}{2} \rho U^{2}}=2\left(\frac{u_{*}}{U}\right)^{2}=2 \frac{\chi^{2}}{\gamma^{2}} \quad \text { and } \quad C_{f}=2 \hat{g}_{0} \operatorname{Re}^{-1}
$$

There is thus a simple relation between $C_{f}$ and $\gamma$, but a more complex relation with Re, because it depends on $\hat{g}_{0}$ and thus on the behaviour of the viscous sublayer (parameter $\psi$ ).

From the fit with the empirical logarithmic profile, we know that this relation between $C_{f}$ and $\gamma\left(\right.$ or $\left.\hat{u}_{*}\right)$ is valid in a fully developed turbulent regime $(\gamma \gg 1)$ but we now make the generalization that Eqs. (49), (50) and (53) can be maintained whatever $\gamma$. The validity of this generalization entirely depends on the value of $\hat{g}_{0}$ that is obtained from the numerical integration of the velocity profile for any given $\gamma$ and $\psi$. In the viscous regime $(\gamma \ll 1)$, the solution of the problem is given by Eqs. (28) and (29), with a constant value of $\hat{g}_{0}$. Eq. (53) then gives the right value of $C_{f}$, proportional to $\mathrm{Re}^{-1}$, and we just need to assume that, even in the viscous flow, there is a small value of $\gamma$, with no influence on the velocity profile, and proportional to $\operatorname{Re}^{1 / 2}$ according to Eq. (51). Inbetween, we can only expect that the formalism will provide an appropriate interpolation. 

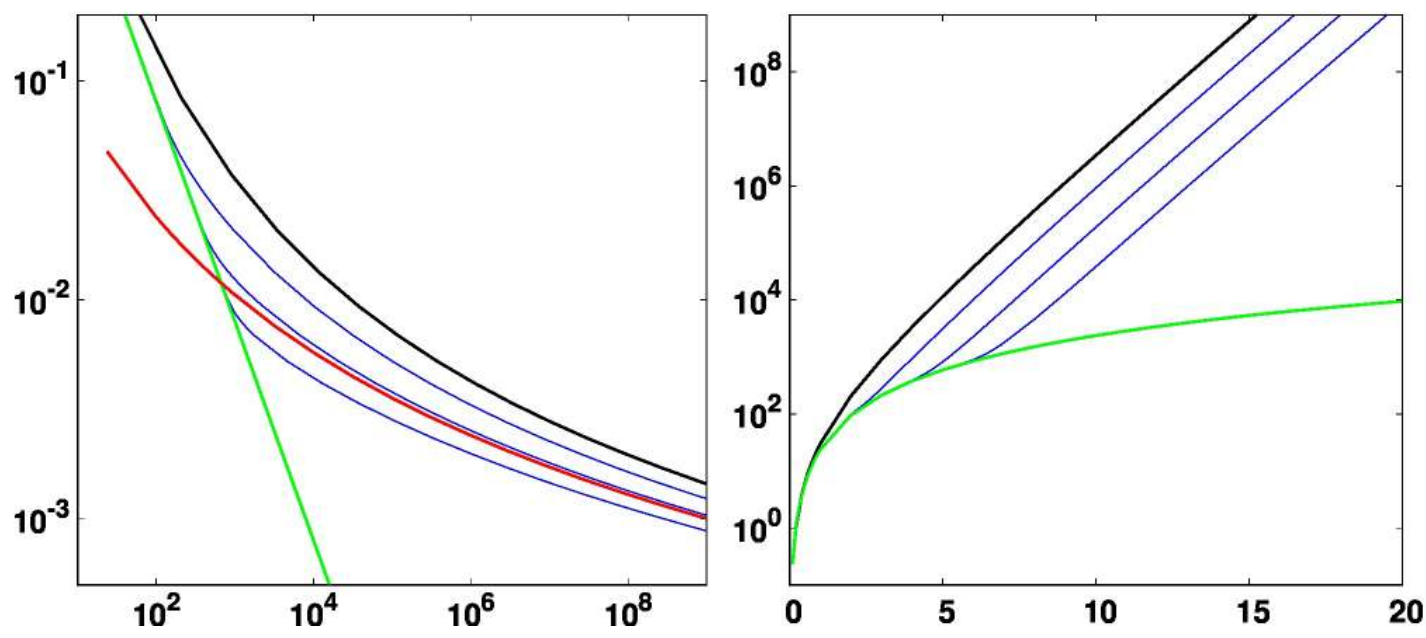

Figure 4: Friction coefficient $\left(C_{f}\right)$ versus Reynolds number (left panel), and Reynolds number versus parameter $\gamma$ (right panel). The solution is shown for $\psi=0$ (black curve), $\psi=3$, $\psi=6$, and $\psi=9$ (blue curves). As $\gamma$ increases, all solutions separate from the linear limit (green curve), to follow the exponential increase of the Reynolds number with $\gamma$. The solution obtained with $\psi=6$ (second blue curve) is closest to the empirical relation between $C_{f}$ and $R e$ in turbulent flows: $\left(2 / C_{f}\right)^{1 / 2}=1 / \chi \ln \left(\operatorname{Re}\left(C_{f} / 2\right)^{1 / 2}\right)+\psi^{+}$(red curve).

Figure 4 (left panel) shows the result obtained for the variation of the friction coefficient $C_{f}$ as a function of the Reynolds number (Re) in the case of the Poiseuille flow. It is shown for $\psi=0$ (black curve), $\psi=3, \psi=6$, and $\psi=9$ (blue curves), and compared to the observed viscous behaviour $C_{f}=8 \mathrm{Re}^{-1}$, valid for small Re (green curve) and to the observed turbulent behaviour (red curve). The latter is obtained from the formula: $\left(2 / C_{f}\right)^{1 / 2}=1 / \chi \ln \left(\operatorname{Re}\left(C_{f} / 2\right)^{1 / 2}\right)+\psi^{+}$, i.e. the same empirical formula used in Orlandi et al. (2015). Consistently with what was obtained for the logarithmic profile in Fig. 3, the best agreement with the observational data is obtained for $\psi=6$ for large Re. The smaller values of $\psi$ overestimates the friction coefficient. On the other hand, for small Re, all curves fit the viscous behaviour, as expected.

Transition to turbulence. At this point, it is worth emphasizing that Fig 4 is the direct result of the minimization of $S[u]$ in Eq. (17) with the boundary conditions in Eq. (24) for various values of $\gamma$ and $\psi$, together with Eqs. (51) and (53) to compute Re and $C_{f}$. As shown above, this single procedure already provides a solution that is quite consistent with observations in the two regions $\operatorname{Re}<10^{2}$ and $\operatorname{Re}>10^{4}$. The transition between the two regimes occurs in a range of Reynolds numbers that is consistent with the observations, but it is very sensitive to $\psi$, i.e. to the extension of the viscous sublayer from both walls towards the interior of the flow. With our simple model of Eq. (45), the flow becomes fully viscous $\left(\hat{g}_{0}=4\right)$ when they join at $\hat{x}=1 / 2$, and becomes turbulent $\left(\hat{g}_{0}>4\right)$ when they disjoin. For $\psi=6$, this occurs for the critical Reynolds number $\operatorname{Re}_{c} \simeq 400$.

In summary, these results suggest that the empirical description of the velocity profile for all Reynolds numbers can be quite faithfully reproduced by the nonlinear dissipation model with parameters $\gamma$ and $\psi$. The characteristics of the flow depending on $\gamma$ only are (i) the shape of the normalized velocity profile, outside of the viscous sublayer (as illustrated in Fig. 1 and 2), and (ii) the friction coefficient $C_{f}$, given by Eq. (53). On the contrary, the Reynolds number is more indirectly related to $\gamma$ (as illustrated in Fig. 4, right panel), because the wall slope $\hat{g}_{0}$, which is exponentially large for $\gamma \gg 1$, is very sensitive to the specific behaviour of the flow in the viscous sublayer (blue curves as compared to the black curve). This is what makes the description of the flow as a function of Re more intricate for large $\gamma$. 


\subsection{Fluctuations}

A more complete study would also use observations to assess uncertainties associated to the velocity profile, as described by the probability distribution in Eq. (4). In presence of turbulence, uncertainties in the macroscopic profile are likely to be larger, because much less unresolved degrees of freedom contribute to the flow. A large uncertainty also means that the mean velocity profile can be somewhat different from the maximum probability velocity profile computed in section 4.1 .
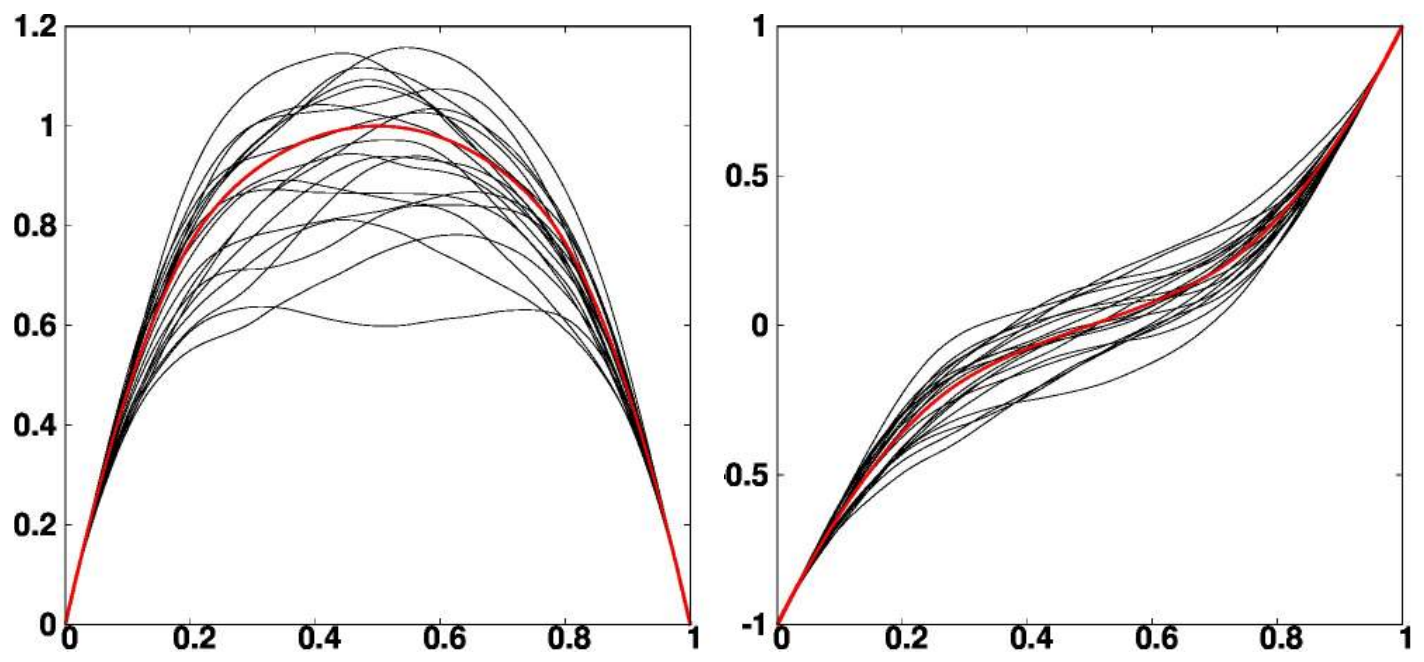

Figure 5: Samples of 20 Poiseuille (left panel) and Couette (right panel) velocity profiles (black curves), drawn from their probability distribution, with parameters $\gamma=5$ and $\psi=6$. Velocity $\hat{u}$ (y-axis) is drawn as a function of $\hat{x}$ (x-axis). They are compared to the maximum probability solution (red curve), with $\operatorname{Re}=825$ (Poiseuille flow) and $\mathrm{Re}=672$ (Couette flow).

To illustrate possible macroscopic fluctuations of the velocity profile, Fig. 5 shows an ensemble of 20 velocity profiles sampled from the probability distribution in Eq. (4), with $S[u]$ given by Eq. (17). They are drawn with parameters $\gamma=5$ and $\psi=6$, and for a given value of the wall slope $\hat{g}_{0}$, corresponding to the maximum probability velocity profile, so that they do not respect the normalization condition on velocity anymore. To display a sufficient spread, the constant $S_{0}$ is here arbitrarily chosen to be $10 \%$ of the minimum value of $S[u]$. In this figure, we see that the sample is quite consistent with the maximum probability velocity profile computed in section 4.1, and that the fluctuations tend to be small close to the wall, but can take various shapes in the interior of the flow.

In practice, this sample was obtained by a direct application of the standard Metropolis/Hastings algorithm (as described in Robert and Casella, 2004). The proposal probability distribution for $\hat{g}$ was a zero-mean Gaussian distribution with a power-spectrum in $1 / \kappa$. The boundary conditions were automatically satisfied by chosing an appropriate basis of trigonometric functions (sine functions equal to zero for $\hat{x}=0$ and $\hat{x}=1$ ).

What is most important to remark here is that the fluctuations associated with dissipation are non-Gaussian and cannot be characterized by their spectrum or covariance only (as in section 3.2), as a result of the non-quadratic form of Eq. (17). This indicates that the system is further away from equilibrium than usually assumed in the description of dissipation in statistical physics (e.g. Landau, and Lifshitz, 1980, chapter 12). 


\section{Turbulent shallow water flow}

As a second example application of the nonlinear dissipation framework introduced in section 2 and 3, we consider here the problem of a two-dimensional flow in a shallow layer, on the surface of a rotating sphere. This is exactly the same problem as in Brankart (2020), with the difference that we introduce the same nonlinear dissipation term as in the previous section. The probability distribution for the flow, described by the stream function $\psi$ or the relative vorticity $\omega=\Delta \psi$ can thus be directly generalized to:

$$
p(\omega) \sim \exp \left[-S(\omega) / S_{0}\right]
$$

with

$$
S(\omega)=\frac{1}{2} \int_{t_{0}}^{t_{1}} \int_{\Omega}\left[\left(\frac{\delta \omega}{\delta t}\right)^{2}+\nu^{2}(\Delta \omega)^{2}+\nu^{2} \gamma^{2}(\nabla \omega \cdot \nabla \omega)^{2}\right] d \Omega d t
$$

where $\Omega$ is the surface of the sphere (with radius $R$ ). In this case, the residual stochastic trend produced by dissipation is what remains once the effects of rotation, advection and external forcing have been taken into account:

$$
\frac{\delta \omega}{\delta t}=\frac{D}{D t}(\omega+f)-\frac{\omega_{F}}{\tau}
$$

where $f=2 \omega_{s} \sin \theta$ is the planetary vorticity, $\omega_{s}$ is the angular velocity of the sphere, and $\theta$ is latitude. As compared to Brankart (2020), we also introduced a forcing term in the form of a vorticity field $\omega_{F}$ and a timescale $\tau$. Eq. (56) means that, in absence of dissipation $(\nu=0$ and $\gamma=0)$ and forcing $\left(\omega_{F}=0\right)$, conservation of potential vorticity $(\omega+f)$ along the fluid parcels trajectories is the most probable solution.

Instationary flows, with a specified initial condition, can then be sampled from the probability distribution (54), as explained in Brankart (2020). However, several differences have been introduced in the conditions applied to the flow. First, the initial condition is here set to zero $\left(\omega_{0}=0\right)$, while the initial condition that was used in Brankart (2020) is here applied as a constant forcing $\omega_{F}$. The relative vorticity can thus be expected to grow with time until the angular momentum supplied by the forcing is equilibrated by dissipation. Second, adimensionalization is here based on $\omega_{F}$ rather than $\omega_{0}$, with a length scale $L / R=0.1$ and a time scale $T=1 / \operatorname{rms}\left(\omega_{F}\right)$. With this metrics, the forcing time scale is set to $\tau / T=1$, and the numerical time step, to $\Delta t / T=0.1$. Third, to avoid spurious inputs of angular momentum from the boundaries, a tight constraint on the value of $\omega$ (with standard deviation 0.05) has been applied along the boundaries, in the region where the advection constraint is undefined.

The result is illustrated in Fig. 6, which compares three instationary flows sampled from (54) with different values of the dissipation parameters $\nu$ and $\gamma$. The first one (left panels) is obtained without the nonlinear dissipation term $(\gamma=0$, as in Brankart, 2020), and with viscosity $\nu=$ $0.2 L^{2} T^{-1}$. In the second one (middle panels), viscosity is divided by 2 to $\nu=0.1 L^{2} T^{-1}$, and a moderate turbulent dissipation is introduced with $\gamma / T=0.1$. In the third one (right panels), turbulent dissipation is increased to $\gamma / T=1$, with a much smaller viscosity: $\nu=0.01 L^{2} T^{-1}$. In these flows, the parameters $\nu$ and $\gamma$ have been tuned to equilibrate the forcing at about the same root mean square relative vorticity. Thus, with a smaller $\nu, \gamma$ must be increased to generate enough dissipation. In the second flow, $\nu$ and $\gamma$ contribute to dissipation at about the same level, while dissipation is mostly due to $\gamma$ in the third flow.

As can be seen in the figure (and even better in the movie provided in the data repository associated with this paper), the nonlinear dissipation term can equilibrate the forcing by producing flows with sharper gradients, and in which the small scales produced by the forcing can survive for a longer time. To some extent, this behaviour is similar to what has beeen obtained 


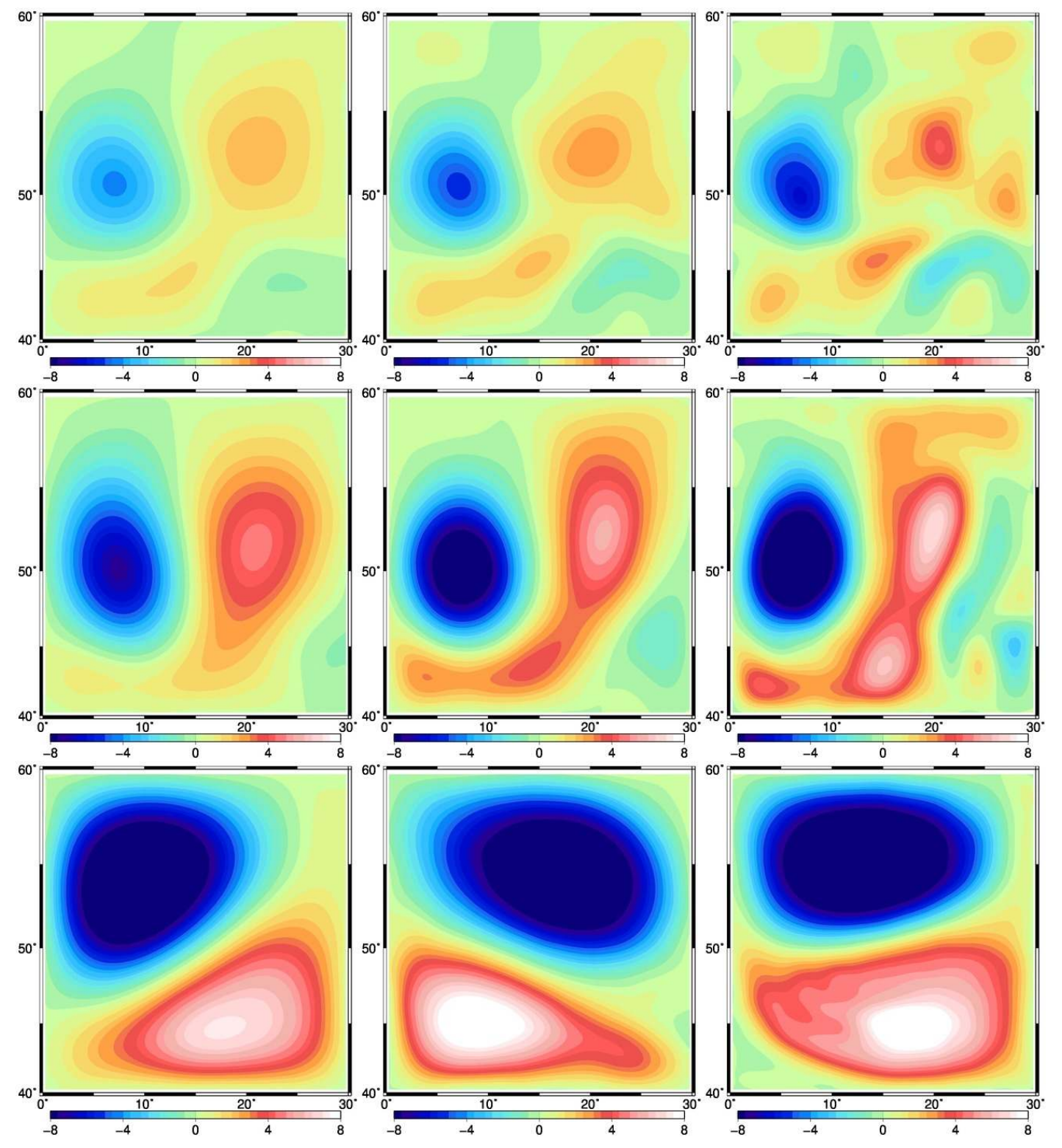

Figure 6: Time evolution of relative vorticity sampled from the probability distribution (54) for different values of the dissipation parameters $\nu$ and $\gamma: \nu=0.2 L^{2} T^{-1}$ and $\gamma=0$ (left panels), $\nu=0.1 L^{2} T^{-1}$ and $\gamma=0.1$ (middle panels), $\nu=0.01 L^{2} T^{-1}$ and $\gamma=1$ (right panels), It is displayed for times: $t / T=2,5$ and 15 (from top to bottom). 
in the previous section for the turbulent boundary layer, and can be thought to be more consistent with the observed behaviour of the atmospheric and oceanic flows. Although preliminary, this result suggests that this nonlinear formulation of turbulent dissipation might provide a way to simulate and forecast geophysical flows, without explicitly resolving the small-scale motions.

In practice, this application being computationally more demanding, the flows displayed in Fig. 6 were obtained using the approximate Metropolis/Hastings algorithm proposed in Brankart (2019), using the settings described in Brankart (2020).

\section{Conclusions}

In summary, in this paper, a physical framework has first been proposed to introduce nonlinear dissipation in the probabilistic description of a macroscopic flow. This has been done by making the probability of the flow decrease with the deviation of the Lagrangian with respect to local equilibrium, and by assuming that this deviation can be computed from the local structure of the macroscopic flow. By construction, this formulation of dissipation is symmetric with respect to the reversal of time, but irreversible flows can be obtained as a result of the asymmetry between the initial and final conditions (as more extensively discussed in Brankart, 2020). In particular, a wide variety of linear dissipation behaviours can be reproduced by adjusting appropriately the spectrum of the stochastic fluctuations in the macroscopic flow.

Second, this reversible and stochastic dynamical framework has been applied to the simulation of turbulent geophysical flows. The results obtained for the turbulent boundary layer show that the main observed characteristics of the flow can be faithfully reproduced using the most simple nonlinear formulation of dissipation, i.e. by introducing the lowest-order nonlinear term, with a constant or piecewise constant phenomenological coefficient. In particular, far enough from the outer conditions and far enough from the wall, the universal logarithmic velocity profile is an analytic solution of the governing equation, and the two empirical constants of the logarithmic profile (the von Karman constant $\kappa$ and the integration constant $\psi^{+}$) are directly involved in the formulation, through constant parameters describing the nonlinear turbulent dissipation. In addition, the behaviour of the friction coefficient as a function of the Reynolds number is correctly simulated by this single model across the viscous and turbulent regimes. Unlike linear dissipation, the nonlinear dissipation term is specific to the reversible formulation of the problem, and has no equivalent as a forward-in-time diffusion equation.

On the other hand, the results obtained with the shallow-water model suggest that the same approach can be used to simulate turbulent dissipation in more complex geometries and more complex flows, and that it might become applicable to produce a probabilistic forecast of geophysical flows. Moreover, if the problem is solved by sampling from a probability distribution, embedding additional conditions from past observations should be straightforward.

More generally, this study suggests that it is possible to construct a reversible probabilistic dynamics that connects observations across time, at a macroscopic level, without explicitly resolving the finest structures of the system. This should require, on the one hand, strengthening the physical justification of the probabilistic formulation using statistical physics. This means for example giving a more concrete physical interpretation of the nonlinear dissipation terms and a method to compute the phenomenological coefficient (with constants $\kappa$ and $\psi$ ) and the spread of the distribution $\left(S_{0}\right)$ from the statistics of the unresolved processes. On the other hand, further comparison to observations in both simple and complex geometry is certainly necessary to measure the reliability and accuracy of the probabilistic model, to identify unknown parameters, and to suggest routes for improvements. 


\section{A Nonlinear relaxation to equilibrium}

In this annex, we focus on the relaxation trajectory of the system towards equilibrium corresponding to the maximum of the probability distribution given in Eq. (4). To simplify the problem, we keep only a quadratic form with the time derivatives and we remove the spatial derivatives. We are thus considering the trajectories $Z_{i}(t)$ minimizing the functional:

$$
S=\int_{t_{0}}^{t_{1}}\left(\sum_{i j} \alpha_{i j} \dot{Z}_{i} \dot{Z}_{j}+\sum_{i j} \beta_{i j} Z_{i} Z_{j}\right) d t
$$

with

$$
\beta_{i j}=\beta_{i j}^{(0)}+\sum_{k l} \beta_{i j k l}^{(1)} Z_{k} Z_{l}+\sum_{k l p q} \beta_{i j k l p q}^{(2)} Z_{k} Z_{l} Z_{p} Z_{q}
$$

to examine in which specific condition they can correspond to the resolution of a first-order forward-in-time relaxation equation.

It is worth mentioning here that we do not really loose generality by not having Laplacian of the $Z_{i}$ in Eq. (57), by comparison to Eq. (5), because they can be replaced by a modal decomposition of the fields, which would just produce more $Z_{i}(t)$ to include in the above sums. Using Eq. (57) rather than Eq. (1) just means that we are here looking at the relaxation problem globally rather than locally. In the main text of the paper, the zeroth order terms of Eq. (57) were ignored because we considered deviations with respect to local equilibrium. In the case of a flow, it is indeed in this framework that the phenomenological coefficients can take sense.

The minimum of the functional in Eq. (57) must satisfy the Euler-Lagrange equations (Gelfand and Fomin, 1964):

$$
\sum_{j} \alpha_{i j} \ddot{Z}_{j}=\sum_{j} \beta_{i j}^{(0)} Z_{j}+2 \sum_{j k l} \beta_{i j k l}^{(1)} Z_{j} Z_{k} Z_{l}+3 \sum_{j k l p q} \beta_{i j k l p q}^{(2)} Z_{j} Z_{k} Z_{l} Z_{p} Z_{q}
$$

We here consider an initial condition problem, with a specified initial condition and a natural final condition:

$$
Z_{i}\left(t_{0}\right)=Z_{i}^{0} \quad \text { and } \quad \sum_{j} \alpha_{i j} \dot{Z}_{j}\left(t_{1}\right)=0 \quad \forall i
$$

This is a system of second-order differential equations that is sometimes reducible to a system of first-order differential equations, as discussed in the following.

Reducibility conditions. Let us consider first the problem of one single variable $Z(t)$ relaxing to equilibrium $(\dot{Z}=0)$ according to the nonlinear first-order differential equation:

$$
\dot{Z}=-Z \sum_{k=0}^{N} \lambda_{k} Z^{2 k} \quad \text { with } \quad Z\left(t_{0}\right)=Z^{0} \quad \text { and } \quad \lambda_{k} \geq 0
$$

By differentiating in time and substituting $\dot{Z}$ using Eq. (61), we obtain the second-order differential equation:

$$
\ddot{Z}=\sum_{k=0}^{N} \sum_{l=0}^{N}(2 k+1) \lambda_{k} \lambda_{l} Z^{2 k+2 l+1}
$$

which must have the same solution as Eq. (61), with the two conditions:

$$
Z\left(t_{0}\right)=Z^{0} \quad \text { and } \quad \dot{Z}\left(t_{1}\right)=0 \quad \text { for } \quad t_{1} \rightarrow \infty
$$


The second condition indeed always corresponds to the final state of Eq. (61), since $\lambda_{k} \geq 0$, $\forall k$. On the other hand, Eq. (62) with conditions (63) has the same form as the Euler-Lagrange equation (59), with conditions (60). They can thus be inferred to describe the minimum of:

$$
S=\int_{t_{0}}^{t_{1}}\left(\dot{Z}^{2}+\sum_{k=0}^{N} \sum_{l=0}^{N} \frac{2 k+1}{k+l+1} \lambda_{k} \lambda_{l} Z^{2 k+2 l+2}\right) d t
$$

which can be rewritten:

$$
S=\int_{t_{0}}^{t_{1}}\left[\dot{Z}^{2}+Z^{2}\left(\sum_{k=0}^{N} \lambda_{k} Z^{2 k}\right)^{2}\right] d t
$$

With all $\lambda_{k} \geq 0$, we see that the minimum of $S$ (which is unique) must be a decreasing function of time for an initial condition problem and an increasing function of time for a final condition problem. Among these two possible solutions of the second-order equation (62), we have to select the first one by applying conditions (63), which is the solution of Eq. (61) providing that the final time is large enough $\left(t_{1} \rightarrow \infty\right)$ compared to the relaxation time scales.

From the above discussion, it can be seen that, for one variable, all relaxation equations that can be put in the form of Eq. (61) correspond to the minimum of a variational principle, providing that only the initial condition is imposed, and that the final condition (for $t_{1} \rightarrow \infty$ ) is left free. But the converse is not true. Not all variational principle in the form of Eq. (57), even for one variable, can be put in the form of a relaxation equation like Eq. (61). For instance, the most simple nonlinear relaxation equation:

$$
\dot{Z}=-\lambda Z-\mu Z^{3}
$$

corresponds to the minimum of:

$$
S=\int_{t_{0}}^{t_{1}}\left[\dot{Z}^{2}+Z^{2}\left(\lambda+\mu Z^{2}\right)^{2}\right] d t
$$

which involves terms in $Z^{2}, Z^{4}$ and $Z^{6}$. But the most simple variational principle:

$$
S=\int_{t_{0}}^{t_{1}}\left(\dot{Z}^{2}+\lambda Z^{2}+\mu Z^{4}\right) d t
$$

does not correpond to any simple first-order forward-in-time relaxation equation. It can thus be expected that this formalism provides more freedom to describe nonlinear relaxation processes.

Reciprocity conditions. To generalize the above discussion to several variables $Z_{i}(t), i=$ $1, \ldots, N$, we start by considering an extension of Eq. (66):

$$
\dot{Z}_{i}=-\sum_{j} \lambda_{i j} Z_{j}-\sum_{j k l} \mu_{i j k l} Z_{j} Z_{k} Z_{l}
$$

where $\mu_{i j k l}$ is invariant for any permutation of the indices $j, k, l$. Repeating then the same operations transforming Eq. (61) to Eq. (62), we obtain the second-order equations:

$$
\ddot{Z}_{i}=\sum_{j k} \lambda_{i j} \lambda_{j k} Z_{k}+3 \sum_{j k l m} \mu_{i j k l} \lambda_{l m} Z_{j} Z_{k} Z_{m}+3 \sum_{j k l m p q} \mu_{i j k l} \mu_{l m p q} Z_{j} Z_{k} Z_{m} Z_{p} Z_{q}
$$

which can be interpreted to be the Euler-Lagrange equation (59) corresponding to the variational principle (57) if: 


$$
\begin{aligned}
\sum_{j} \lambda_{i j} \lambda_{j k} & =\sum_{j} \alpha_{i j}^{-1} \beta_{j k}^{(0)} \\
2 \sum_{l} \mu_{i j k l} \lambda_{l m} & =\sum_{l} \alpha_{i l}^{-1} \beta_{l j k m}^{(1)} \\
\sum_{l} \mu_{i j k l} \mu_{l m p q} & =\sum_{l} \alpha_{i l}^{-1} \beta_{l j k m p q}^{(2)}
\end{aligned}
$$

In Eqs. (71) to (73), the invariance of $\alpha$ and $\beta$ with respect to any permutation of their indices imposes a stringent constraint on the possible relaxation coefficients $\lambda_{i j}$ and $\mu_{i j k l}$. This constraint applies to the linear coefficients $\lambda_{i j}$ alone through Eq. (71), and to the nonlinear coefficients through Eqs. (72) and (73).

This means that, to be acceptable, the coefficients $\lambda_{i j}$ and $\mu_{i j k l}$ of the first-order forward-intime relaxation in Eq. (69) are subjected to reciprocity conditions resulting from the symmetry of $\alpha$ and $\beta$ in Eq. (57). In the linear case, this imposes for instance that there cannot be an effect of variable $Z_{2}$ on the relaxation of variable $Z_{1}\left(\lambda_{12} \neq 0\right)$ without a reciprocal effect of $Z_{1}$ on $Z_{2}\left(\lambda_{21} \neq 0\right)$. This constraint on the linear coefficients $\lambda_{i j}$ in Eq. (71) is very similar to Onsager's reciprocity conditions, except that it is expressed in terms of the square of $\lambda_{i j}$, rather than $\lambda_{i j}$, which results from assuming a second-order time evolution equation rather than a first-order relaxation equation. In the nonlinear case, a similar reciprocity of effects applies to the coefficients $\mu_{i j k l}$, as expressed by Eqs. (72) and (73), in the particular case of the nonlinear relaxation described by Eq. (69). More generally, for any system described by Eq. (57), even if it is not reducible to a first-order forward-in-time relaxation equation, the reciprocity of effects among the $Z_{i}$ directly results from the symmetry of $\alpha$ and $\beta$, which is imposed by the formulation of the problem.

\section{B Evolution equation for velocity}

An equation for velocity with nonlinear dissipation terms was used in section 4 to describe a onedimensional stationary flow. The purpose of this annex is to generlaize this to three-dimensional instationary flows. In the framework of section 2, we have thus three macroscopic fields $Z_{i}$, corresponding to the three components of velocity $\mathbf{u}$. In this case, the residual stochastic trend produced by dissipation in an incompressible flow $(\nabla \cdot \mathbf{u}=0)$ can be written:

$$
\frac{\delta \mathbf{u}}{\delta t}=\frac{D \mathbf{u}}{D t}+2 \boldsymbol{\Omega} \times \mathbf{u}+\frac{1}{\rho} \nabla p
$$

where the effect of advection, Coriolis force (with angular velocity $\boldsymbol{\Omega}$ ) and pressure gradient (with pressure $p$ and density $\rho$ ) have been taken into account. Eq. (74) is just a rewriting of the stochastic Euler equation, which was used as a starting point in the Annex A of Brankart (2020) in the more specific case of a linear dissipation.

According to Eqs. (3) and (4), the maximum probability flow can then be obtained by minimizing:

$$
S(\mathbf{u})=\int_{t_{0}}^{t_{1}} \int_{\Omega}\left[\frac{\delta \mathbf{u}}{\delta t} \cdot \frac{\delta \mathbf{u}}{\delta t}+\nu^{2} \Delta \mathbf{u} \cdot \Delta \mathbf{u}+\gamma^{2}(\nabla \mathbf{u}: \nabla \mathbf{u})^{2}\right] d \Omega d t
$$

where we kept the same nonlinear term as in section 4. Isotropy was assumed, so that the coefficient of the first term can be rescaled to 1, and only one free parameter remains for each of the other terms $(\nu$ and $\gamma)$.

Proceeding then as in the annexes A and B of Brankart (2020), we directly obtain the corresponding Euler-Lagrange equation: 


$$
\left(\frac{D}{\delta t}-2 \boldsymbol{\Omega} \times-\nabla \mathbf{u} \cdot\right)\left(\frac{D \mathbf{u}}{\delta t}+2 \boldsymbol{\Omega} \times \mathbf{u}+\frac{1}{\rho} \nabla p\right)=\nu^{2} \Delta \Delta \mathbf{u}-2 \gamma^{2} \nabla \cdot[(\nabla \mathbf{u}: \nabla \mathbf{u}) \nabla \mathbf{u}]
$$

Without the nonlinear dissipation term (with $\gamma=0$ ), this can be viewed as a rewriting of the Navier-Stokes equations, in which diffusion can occur symmetrically in the two directions of time. In this symmetric framework, it is then possible to introduce nonlinear dissipation terms, which are not always reducible to a forward-in-time dissipation operator (as explained in Annex A).

Acknowledgments. All codes necessary to reproduce the example application described in this paper are openly available from the repository 'flowsampler' at github.com/brankart, while data can be found in the repository 'flowsampler-data'. This work benefited from support from CNES through the OST/ST Science Team. The calculations were performed using HPC resources from GENCI-IDRIS (grant A6-011279).

\section{References}

Abramowitz, M. and I. A. Stegun, 1970: Handbook of Mathematical Functions with Formulas, Graphs, and Mathematical Tables. New York: Dover Publications, Ninth printing.

Brankart J.-M., 2019: Implicitly Localized MCMC Sampler to Cope With Non-local/Non-linear Data Constraints in Large-Size Inverse Problems. Front. Appl. Math. Stat., 5:58.

Brankart J.-M., 2020: Sampling geophysical flows from a dynamically constrained probability distribution, JAMES, submitted.

Delhez, E.J.M. and E. Deleersnijder, 2007: Overshootings and spurious oscillations caused by biharmonic mixing, Ocean Modelling, 17, 183-198..

Gelfand, I. M. and S. V. Fomin, 1964: Calculus of variations. Englewood Cliffs, Prentice-Hall.

Hinze J.O., 1959: Turbulence. MacGraw Hill, Oxford, first edition.

Landau, L. D. and E. M. Lifshitz, 1980. Statistical Physics. Vol. 5 (3rd ed.). ButterworthHeinemann.

Orlandi, P., M. Bernardini and S. Pirozzoli, 2015. Poiseuille and Couette flows in the transitional and fully turbulent regime. J. Fluid Mech., 770, 424-441.

Robert, C. and P. Casella, 2004: Monte Carlo Statistical Methods. Springer, 645 pp.

Schlichting H., 1979: Boundary layer theory. MacGraw Hill Book Comp., seventh edition. 